\title{
Technologieinstitut und Forschungsrat: Zwei Instrumente europäischer Exzellenz
}

\author{
Waldemar Hummer*
}

\section{Die ,Innovationskluft' ${ }^{\star}$ zwischen der EU und den USA}

„Given its tradition of scientific excellence and abundance of scientific talent, Europe should be leading the world in pioneering research (...). However Europe has not only lost its leadership position in many areas of science to the US, it is also in danger of being outgunned by the rising giants of Asia - India and China."1

Im Wissen um diese Herausforderung plant die Europäische Kommission, einen „Europäischen Forschungsraum“2 zu errichten und versucht, die „Innovationskluft“ zwischen Europa und den USA, aber auch Japan, China und Indien zu verringern. Dabei sind die Ausgangsvoraussetzungen keinesfalls günstig. Wie die Ratsvorsitzende, Angela Merkel, auf der offiziellen Gründungskonferenz des ,Europäischen Forschungsrates ‘ (European Research Council, EFR/ERC) am 27. Februar 2007 in Berlin anmerkte, ,fehlen der EU rund 700.000 Forscher“, noch dazu, wo die EU ein Netto-Exporteur von Wissenschaftlern sei. ${ }^{3}$ In Deutschland ist dabei vor allem ein Mangel an Ingenieuren und Naturwissenschaftlern zu beklagen. Auch schaffen in der Bundesrepublik nur 20,6 Prozent eines Altersjahrganges einen Hochschulabschluss, während dieser Prozentsatz in den 30 wichtigsten Industrienationen im Schnitt bei 34,8 Prozent liegt. In Deutschland erwerben auch nur 38,8 Prozent eines Altersjahrgangs mit einem Abschluss im Sekundarbereich II die Hochschulreife, der Schnitt aller Industriestaaten liegt gemäß dem jüngsten Bildungsbericht der OECD aber bei 67,7 Prozent. ${ }^{4}$

Ganz allgemein betrachtet würde die bisherige EU-25 nach neuesten Untersuchungen mehr als 50 Jahre benötigen, um die USA in puncto Innovationsleistung einzuholen. 2004 betrugen die Ausgaben für Forschung und Entwicklung (F\&E) in der EU 1,90 Prozent des Bruttoinlandsprodukts und waren damit deutlich niedriger als in den USA (2,59 Prozent) und in Japan (3,15 Prozent). Von den 500 weltweit besten Universitäten befinden sich zwar 205 gegenüber 198 in den USA - in Europa, es zählen jedoch nur 2 europäische Universitäten zu den besten 20 - wiederum gegenüber 17 in den USA. Auch die kritische Konzentration personeller, finanzieller und materieller Ressourcen erfolgt in den USA gezielter als in Europa: Während in der EU 2.000 Universitäten forschungsaktiv sein möchten, fließen in den USA 95 Prozent der Bundesfördermittel für den Universitätsbereich in knapp 200 der insgesamt 3.300 Hochschulen - ein eindeutiger Hinweis auf eine gezielte Förderung von Eliteuniversitäten.

* O. Univ.-Prof. Dr. Dr. Dr. Waldemar Hummer, Institut für Europarecht und Völkerrecht der Universität Innsbruck.

1 Europäischer Forschungsrat: Supporting the next generation of top research leaders in Europe, European Research Council opens for business, 07.02.2007, abrufbar unter: http://erc.europa.eu/index.cfm?fuseaction=nwev.newsReader\&year=2007 (letzter Zugriff: 08.03.2007).

2 Vgl. Mitteilung der Kommission an den Rat, das Europäische Parlament und den WSA „Hin zu einem europäischen Forschungsraum“, $\operatorname{KOM}(2000) 6$ endgültig; hinsichtlich der Durchführungsmaßnahmen vgl. KOM(2001) 215 endgültig; $\operatorname{KOM(2001)~} 331$ endgültig; KOM(2001) 346 endgültig; KOM(2001) 549 endgültig.

3 Europäischer Forschungsrat: Supporting the next generation of top research leaders in Europe.

4 Angela Merkel: In der EU fehlen 700000 Forscher, in: Yahoo! Nachrichten, 27.02.2007; http://de.news. yahoo.com/270207/3/merkel-eu-fehlen-700-nbsp-000-forscher.html (letzter Zugriff: 27.02.2007); OECD (Hrsg.): Bildung auf einen Blick/Education at a Glance. OECD-Indikatoren 2006, S. 51, 57 und 66. 
Die Hauptschwäche Europas liegt darin, dass die drei Seiten des ,Wissensdreiecks ‘ Ausbildung, Forschung und Innovation - strukturell nicht miteinander verbunden sind, sodass zum einen die Praxis die Ausbildung und Forschung zu wenig stimuliert und zum anderen die praktische Vermarktung von Forschungsergebnissen noch zu wünschen übrig lässt. Europa kämpft noch immer damit, F\&E-Ergebnisse effektiv in Geschäftsmöglichkeiten umzusetzen, personelle, finanzielle und materielle Ressourcen in der Forschung und im Hochschulbereich zu konzentrieren, eine von Innovation und unternehmerischer Initiative getragene Kultur in Forschung und Ausbildung zu fördern sowie neue, an die heutigen Anforderungen angepasste Organisationsmodelle zu entwickeln. Diese Kluft manifestiert sich zum Beispiel darin, dass die Zahl der im privaten Sektor beschäftigten Forscher in der EU einerseits und in den USA und Japan andererseits völlig unterschiedlich ist: Während in den USA 80 Prozent aller Forscher und in Japan immerhin 67 Prozent in der Privatwirtschaft tätig sind, arbeitet dagegen in der EU nur knapp die Hälfte aller Forscher (47 Prozent) in privaten Unternehmen.

Im März 2000 legte der Europäische Rat in der ,Lissabon-Strategie ${ }^{65}$ das Ziel fest, die Union innerhalb von zehn Jahren, das heißt bis 2010, zum ,wettbewerbsfähigsten und dynamischsten wissensbasierten Wirtschaftsraum der Welt" zu machen. Dazu wären die Investitionen in Forschung und technologische Entwicklung zu erhöhen und der Investitionsrückstand gegenüber den größten Konkurrenten Europas aufzuholen. Bis 2010 sollten daher die Investitionen von 1,9 Prozent auf 3 Prozent des BIP der EU steigen, wovon zwei Drittel von Unternehmen getragen werden sollen. Um dieses Ziel zu erreichen, müssten der öffentliche und der private Sektor ihre Forschungsinvestitionen in Europa um durchschnittlich 6,5 Prozent beziehungsweise 9,5 Prozent pro Jahr erhöhen. ${ }^{6}$

Während Europa aber auf dem Gebiet der Innovation neuerdings zu den USA aufzuschließen beginnt, bleibt es auf dem Sektor der Spitzenforschung weiterhin zurück. Gemäß dem neuesten Innovationsanzeiger der $\mathrm{EU}^{7}$ schließt sich die Innovationslücke zwischen der EU und den USA seit 2003 nach und nach. In diesem Zusammenhang sind vor allem die nordischen Staaten Schweden, Dänemark und Finnland führend, die sich sogar im weltweiten Vergleich an die Spitze gesetzt haben. Auch beim lebenslangen Lernen sind diese Staaten führend, wobei die Schweden mit 35 Prozent der Bevölkerung zwischen dem 25. und dem 65. Lebensjahr weltweit führend sind, während der gesamteuropäische Durchschnitt bei 11 Prozent liegt.

Trotzdem gelingt es Europa nach wie vor nicht, Forschungs- und Entwicklungsergebnisse unmittelbar in konkrete unternehmerische Aktivitäten umzusetzen. Ausbildung, Forschung und Innovation müssen daher stärker miteinander verknüpft werden, damit sie einen größeren Beitrag zu Wirtschaftswachstum, Beschäftigung und sozialem Zusammenhalt leisten können. Angesichts dieser Defizite hat die Kommission im Frühjahr 2005 als wichtigen Beitrag zur Stärkung von Wachstum und Wettbewerbsfähigkeit in Europa die Einrichtung eines ,Europäischen Technologieinstituts‘ vorgeschlagen. Auf dem Sektor der Spitzenfor-

5 Europäischer Rat (Lissabon): Schlussfolgerungen des Vorsitzes, 23./24. März 2000.

6 Vgl. Europäische Kommission: In die Forschung investieren: Aktionsplan für Europa, KOM(2003) 226 endgültig.

7 Maastricht Economic Research Institute on Innovation and Technology (MERIT)/Joint Research Centre of the European Commission: European Innovation Scoreboard (2007), abrufbar unter: http://www.proinno-europe.eu/inno-metrics.html; vgl. dazu: Die Innovationslücke zwischen der EU und den USA schließt sich weiter, IP/07/221, 22.02.2007; European Innovation scoreboard 2006: Summary of the situation in the 27 Member States, MEMO/07/74, 22.02.2007. 
schung (, frontier research $)^{8}$ hingegen hinkt Europa gegenwärtig den USA dramatisch nach. Nimmt man allein die Verleihung von Nobelpreisen als Maßstab für außergewöhnliche wissenschaftliche Leistungen, so spielten in den Jahren 1901 bis 1932 europäische Forschungsinstitute eine führende Rolle: In dieser Periode stellten 35 deutsche, 20 britische und 15 französische Institute Nobelpreisträger, während es lediglich acht amerikanische, zwei kanadische und ein russisches Institut waren. In der Periode 1966 bis 2006 kamen hingegen 216 (!) Nobelpreisträger aus amerikanischen Instituten, während (nur) 32 aus britischen, 16 aus deutschen und elf aus französischen wissenschaftlichen Einrichtungen kamen. Japan stellte in diesen 40 Jahren fünf und Russland vier Nobelpreisträger. ${ }^{9}$

Betrachtet man die Zitationsrate wissenschaftlicher Veröffentlichungen (Quotation Index) als Indikator für Spitzenforschung, so betrug diese von Wissenschaftlern aus der EU-15 lediglich zwei Drittel jener der USA. Wenngleich sich auch diese Rate in der Zwischenzeit zugunsten der europäischen Forscher gebessert hat, ist die Position US-amerikanischer Forscher nach wie vor extrem stark. Obwohl US-Forscher nur etwa ein Drittel aller wissenschaftlichen Publikationen weltweit veröffentlichen, erhalten sie mehr als die Hälfte (!) aller Zitate und stellen nicht weniger als zwei Drittel der weltweit am häufigsten zitierten Arbeiten und Wissenschaftler. Aus europäischer Sicht gilt es also, diesem Trend entgegenzusteuern.

Die Europäische Kommission hat deshalb im Zuge der Ausarbeitung des Siebten Forschungsrahmenprogramms $(2007-2013)^{10}$ im September 2005 die vier wichtigsten spezifischen Programme vorgelegt, innerhalb derer das Unterprogramm ,Ideen ' die Einrichtung eines Europäischen Forschungsrates als unabhängige Einrichtung zur Förderung der Spitzenforschung vorschlug. Dieser stellt die erste europäische Einrichtung zur Förderung der Pionierforschung in allen wissenschaftlichen und akademischen Bereichen dar und ist damit eine sinnvolle Ergänzung zum vorstehend erwähnten Europäischen Technologieinstitut.

Damit gilt es, nachstehend sowohl das Europäische Technologieinstitut als auch den Europäischen Forschungsrat kurz darzustellen und in der Folge auch deren Tauglichkeit zur Behebung der ,Innovations- und Technologie-Kluft ' zwischen Europa und anderen führenden Industrienationen zu untersuchen.

\section{Das ,Europäische Technologieinstitut'}

Im Rahmen der Halbzeitüberprüfung der vorerwähnten Lissabon-Strategie präsentierte die Europäische Kommission in ihrem Frühjahrsbericht 2005 folgende neue Initiative: „Um unser Bekenntnis zum Wissen als dem Schlüssel zu Wachstum zu unterstreichen, schlägt die

8 „However in today”s terms the distinction between „,basis“ and ,,applied“ research has become blurred, due to the fact that emerging areas of science and technology often cover substantial elements of both. As a result, the term ,frontier research" was coined for ECR activities since they will be directed towards fundamental understanding, at the „frontier“ of knowledge.“ ECR (Hrsg.): Supporting the next generation of top research leaders in Europe; vgl. ERC (Hrsg.): ERC Mission, The European Research Council: Bringing great ideas to life, abrufbar unter: http://erc.europa.eu/index.cfm?fuseaction=page.display\&topicID=12 (letzter Zugriff: 12.03.2007).

9 ERC-Auftaktveranstaltung, Berlin 27.-28. Februar 2007, Story Line „Noble Prices“, Die Nobelpreise zurück nach Europa bringen, abrufbar unter: http://erc.europa.eu/pdf/bring_the-nobles-back-to-europe_de.doc (letzter Zugriff: 12.03.2007).

10 Beschluss 1982/2006/EG des Europäischen Parlaments und des Rates vom 18. Dezember 2006 über das Siebte Rahmenprogramm der EG für Forschung, technologische Entwicklung und Demonstration (2007-2013), Amtsblatt 2006, Nr. L 412, S. 1; Beschluss des Rates vom 18. Dezember 2006 über das Siebte Rahmenprogramm der Europäischen Atomgemeinschaft (Euratom) für Forschungs- und Ausbildungsmaßnahmen im Nuklearbereich (2007-2013), Amtsblatt 2006 Nr. L 391, S. 19. 
Kommission die Schaffung eines Europäischen Technologieinstituts vor, das zu einem Magneten für die besten Köpfe, Ideen und Unternehmen aus der ganzen Welt werden soll“". Dieses Europäische Technologieinstitut (European Technology Institute, ETI) soll zu einer Vernetzung und Bündelung europäischer Spitzenleistungen in ,Wissens- und Innovationsgemeinschaften " führen und wäre damit weltweit - wenn überhaupt - nur mit dem Massachusetts Institute of Technology (MIT) vergleichbar, wenngleich dieses auch viel stärker zentralisiert und radiziert ist. Von Insidern wird auch ein Vergleich mit dem Howard Hughes Medical Institute in den USA gezogen. ${ }^{12}$

Die Kommission leitete in der Folge einen Reflexionsprozess ein, auf dessen Grundlage sie am 22. Februar 2006 eine erste Mitteilung ${ }^{13}$ vorlegte. Der Europäische Rat erkannte in den Schlussfolgerungen des Vorsitzes zu seiner Tagung vom 23./24. März 2006 an, dass ein „Europäisches Technologieinstitut ein wichtiger Schritt sein wird, um das bisher fehlende Bindeglied zwischen Hochschulen, Forschung und Innovation zu schaffen" und forderte die Kommission auf, einen entsprechenden Vorschlag für weitere Schritte vorzulegen. ${ }^{14}$ Am 8. Juni 2006 veröffentlichte die Kommission eine zweite Mitteilung, die einige spezifische Fragen in Bezug auf die vorgeschlagene Struktur und Arbeitsweise des ETI beleuchtete. Auf der Tagung des Europäischen Rates vom 15./16. Juni 2006 bekräftigten die Staats- und Regierungschefs, dass ,das Europäische Technologieinstitut, das mit bestehenden nationalen Einrichtungen zusammenarbeiten wird, neben anderen Maßnahmen, die Vernetzung und Synergien zwischen herausragenden Forschungs- und Innovationsgemeinschaften in Europa fördern, ein wichtiger Schritt sein wird, um die Lücke zwischen Hochschulbereich, Forschung und Innovation zu schließen". ${ }^{15}$ Uno actu forderte der Europäische Rat die Kommission auf, ,einen förmlichen Vorschlag für die Errichtung dieses Instituts im Herbst 2006 nach Anhörung des ,Europäischen Forschungsrates ', der Mitgliedstaaten und anderer interessierter Kreise" vorzulegen.

Dementsprechend legte die Kommission am 13. November 2006 einen auf Art. 157 Abs. 3 EGV gestützten „Vorschlag für eine Verordnung des Europäischen Parlaments und des Rates zur Einrichtung des Europäischen Technologieinstituts"16 vor, der die Errichtung eines ETI als unabhängige Einrichtung (Art. 7) mit eigener Rechtspersönlichkeit (Art. 10) vorsieht.

Die deutsche Präsidentschaft und die Europäische Kommission haben kürzlich ein zweistufiges Verfahren zur Errichtung des ETI vorgeschlagen, in dem es zwei formelle Beschlüsse des Rates und des Europäischen Parlaments geben soll: Zunächst sollte im Jahr 2007 eine erste Verordnung erlassen werden, mittels derer das ETI provisorisch gegründet werden würde. In dieser provisorischen Phase sollte der Verwaltungsrat ein detailliertes Konzept ausarbeiten und eine erste, nachstehend noch näher darzustellende ,Wissens- und Innovationsgemeinschaft' als Pilotprojekt gründen. Im Jahr 2008 sollte dann die Europäische Kommission gemeinsam mit dem Verwaltungsrat dem Rat und dem Europäischen Par-

11 Europäische Kommission: Mitteilung für die Frühjahrstagung des Europäischen Rates, KOM(2005) 24 endgültig, S. 23.

12 Vgl. „Es geht nur um gute Wissenschaft“ - Interview mit dem Generalsekretär des Europäischen Forschungsrates Ernst-Ludwig Winnacker, in: Der Tagesspiegel, 27.02.2007.

13 Europäische Kommission: Umsetzung der erneuerten Partnerschaft für Wachstum und Arbeitsplätze - Ein Markenzeichen für Wissen: Das Europäische Technologieinstitut, $\operatorname{KOM}(2006) 77$ endgültig.

14 Europäischer Rat: Schlussfolgerungen des Vorsitzes, 23./24. März 2006, S. 7.

15 Europäischer Rat: Schlussfolgerungen des Vorsitzes, 15./16. Juni 2006, S. 9.

16 Europäische Kommission: Vorschlag für eine Verordnung des Europäischen Parlaments und des Rates zur Einrichtung des Europäischen Technologieinstituts, SEK(2006)1313; SEK(2006)1314; 2006/0197 (COD). 
lament ein definitives Errichtungskonzept für das ETI vorlegen. Mit der Annahme dieses Vorschlages wäre dann das ETI formell gegründet.

Mehrere Mitgliedstaaten wetteifern hinsichtlich der Lokalisierung der zentralen Verwaltungsstruktur des ETI: so verfolgt Österreich nach wie vor das Ziel, diese in Österreich anzusiedeln. Neben Österreich haben aber auch Polen und Ungarn sowie drei deutsche Länder Bayern, Hessen und Nordrhein-Westfalen - ihr Interesse an einer Radizierung des ETI auf ihrem Hoheitsgebiet angemeldet. Für die Zeit der provisorischen Phase wird das Sekretariat des ETI aber wohl sinnvollerweise in Brüssel angesiedelt werden.

\section{Zielsetzung und Aufgaben}

Das ETI ist als eine Einrichtung konzipiert, die in den Bereichen Ausbildung, Forschung und Innovation tätig werden und auf eine Integration dieser drei Bereiche hinarbeiten soll. Das ETI, das von seiner Struktur her darauf angelegt ist, diese miteinander kommunizierenden Bereiche zusammenzuführen, soll zu einem Exzellenzzentrum werden. Mit seiner Errichtung soll unter anderem Folgendes erreicht werden: Minderung der Zersplitterung des europäischen Wissenssektors, Schaffung neuer Referenzmodelle auf der Grundlage von Exzellenz, Integration der wirtschaftlichen und innovatorischen Dimension in Forschung und Ausbildung sowie Aufholung des Innovationsrückstandes.

Seine Aufgabe würde dementsprechend vor allem darin bestehen, die talentiertesten Studenten, Wissenschaftler und Fachkräfte in Europa anzuziehen und sie in der Folge für die Zusammenarbeit mit führenden Unternehmen bei Entwicklung, Management und Nutzbarmachung von Wissen und Forschung zu gewinnen. Dem privaten Sektor würde das ETI ermöglichen, die Qualität seiner Beziehungen zum Ausbildungsbereich sowie zur Forschung mit dem Ziel zu optimieren, bessere Möglichkeiten für die Vermarktung von Produkten zu erschließen und den Austausch zwischen den verschiedenen Akteuren zu intensivieren. Damit würde dem ETI auch Zugang zu privatem Forschungskapital eröffnet werden. Durch diese Einbindung von Universitäten, Forschungseinrichtungen und Unternehmen dürfte das ETI einen speziellen Vorteil gegenüber traditionell organisierten Universitäten haben. Schließlich würde mit der Errichtung des ETI eine Konzentration der vorhandenen Ressourcen ermöglicht, sodass man höchsten Ansprüchen an Exzellenz genügen könnte.

\section{Organisationsform und Arbeitsweise}

Was die Organisationsform des ETI betrifft, so stellte die Kommission zunächst vier Optionen zur Diskussion: eine einzige Einrichtung, ein kleines Netzwerk, ein großes Netzwerk sowie ein ,ETI-Label' und regte darüber eine öffentliche Konsultation an. Im Zuge dieser Konsultationen befürwortete zwar die Mehrheit eine der beiden Netzwerklösungen, ein Viertel der Befragten sprach sich jedoch für eine einzige Einrichtung aus - die allerdings nicht mit einem einzigen Standort verwechselt werden darf.

Unter Berücksichtigung dieser Anregungen schlug die Kommission in ihrem Verordnungs-Vorschlag ${ }^{17}$ das Modell einer autonomen Organisation vor, die die besten Ressourcen auf Gemeinschaftsebene zusammenziehen und im Rahmen von flexiblen, trans- und interdisziplinären ,Wissens- und Innovationsgemeinschaften“ (,Knowledge and Innovation Communities“, KIC) koordinieren soll. Ein solches ,integriertes ETI“18 wäre für begabte Studenten und hochkarätig Forschende attraktiv, würde modernste trans- und interdisziplinäre Innovation und Forschung fördern und wäre auch im weltweiten Wettstreit um private

17 Vgl. SEK(2006) 1313 (Fn. 16).

18 Vgl. SEK(2006) 1313 (Fn. 16), S. 8. 
und öffentliche Mittel erfolgreich. Dazu soll das ETI zahlreiche Partnerschaften mit bestehenden Universitäten, Forschungszentren und Unternehmen (,Partnereinrichtungen') eingehen und diese zu ,Wissens- und Innovationsgemeinschaften " vernetzen, die wiederum selbst Teil des ETI sind. Das ETI wird damit seiner Tätigkeit im Rahmen von, Wissens- und Innovationsgemeinschaften " nachgehen. Diese KIC basieren auf einem integrierten Netzwerkkonzept, gehen jedoch darüber noch hinaus: Es soll sich dabei um integrierte Partnerschaften oder Joint Ventures - unabhängig vom noch festzulegenden genauen Rechtsstatus derselben - zwischen der Privatwirtschaft, der Forschung und herausragenden Teams von Forschungsinstituten und Hochschulen handeln, die ihre personellen, finanziellen und materiellen Ressourcen poolen, um die Schaffung, Verbreitung und Anwendung neuer wissensbasierter Produkte zu fördern.

Diese ,Wissens- und Innovationsgemeinschaften“ (Art. 5 Verordnungs-Vorschlag) würden als Partner-Fachabteilungen und Teams in Universitäten, Forschungseinrichtungen und Unternehmen bestehen und sollen verschiedene Ressourcen - wie Infrastrukturen, Personal und von öffentlicher und privater Seite bereitgestellte finanzielle Ressourcen - gemeinsam nutzen. Obwohl die physischen Ressourcen weiterhin an unterschiedlichen Standorten angesiedelt wären, würde jede ,Gemeinschaft' jedoch als integriertes Ganzes funktionieren. Die ,Wissens- und Innovationsgemeinschaften "würden vom Lenkungsausschuss des ETI im Wege eines wettbewerbsbasierten Verfahrens ausgewählt werden und wären diesem gegenüber auch rechenschaftspflichtig.

\section{Institutionelle Struktur}

Gemäß Art. 8 Verordnungs-Vorschlag in Verbindung mit der ,Satzung' des ETI im Anhang des Verordnungs-Vorschlages ${ }^{19}$ werden folgende vier Organe im ETI eingerichtet.

Ein Verwaltungsrat, der sich sowohl aus ernannten (ein ausgewogenes Verhältnis von unternehmerischer Erfahrung und akademischer Forschungserfahrung widerspiegelnden), als auch aus gewählten (aus den Reihen des Innovations-, Forschungs-, Lehr-, Technik- und Verwaltungspersonals, der Studierenden und Doktoranden des ETI und der KIC) Mitgliedern zusammensetzt.

Es gibt 15 ernannte Mitglieder, deren Amtszeit sechs Jahre beträgt, die nicht verlängerbar ist. Sie werden von der Europäischen Kommission auf Vorschlag eines Findungsausschusses ernannt, der seinerseits wieder aus vier hochrangigen Experten besteht, die von der Kommission ernannt werden. Alle zwei Jahre wird ein Drittel der ernannten Mitglieder ausgewechselt. Die Mitglieder, deren Amtszeit nach dem zweiten bzw. nach dem vierten Jahr nach der ersten Bestellung des Verwaltungsrates endet, werden per Los bestimmt. Es gibt vier gewählte Mitglieder, deren Amtszeit drei Jahre beträgt, die einmal verlängert werden kann. Ihre Amtszeit endet, wenn sie das ETI oder eine KIC verlassen. Die Bedingungen und Modalitäten für die Wahl und Ersetzung der gewählten Mitglieder werden auf Vorschlag des Direktors vom Verwaltungsrat genehmigt, bevor die erste KIC ihre Tätigkeit aufnimmt. Dieser Mechanismus soll eine angemessene Repräsentativität - daher werden die gewählten Mitglieder auch ,repräsentative“ Mitglieder genannt - sicherstellen und die Entwicklung des ETI und der KIC berücksichtigen.

Der Verwaltungsrat tritt mindestens drei Mal jährlich zusammen und wählt seinen Vorsitzenden, dessen Amtsdauer drei Jahre beträgt, aus den Reihen der ernannten Mitglieder. Der Verwaltungsrat beschließt in der Regel mit einfacher Mehrheit seiner Mitglieder.

$19 \operatorname{SEK}(2006) 1313$ (Fn. 16), $26 \mathrm{ff}$. 
Der Verwaltungsrat ist gemäß Art. 8 Abs. 1 lit. a) Verordnungs-Vorschlag in Verbindung mit Art. 2 Satzung vor allem zuständig für: Festlegung der strategischen Prioritäten des ETI sowie der wichtigsten thematischen Bereiche seiner Tätigkeit; Zuweisung von Ressourcen an die einzelnen ,Wissens- und Innovationsgemeinschaften '; Gewährleistung von Exzellenz innerhalb des ETI; Organisation der Auswahl, Kontrolle und Evaluierung der, Wissens- und Innovationsgemeinschaften“; Koordination zwischen den ,Wissens- und Innovationsgemeinschaften'; Zuweisung der Mittel an die ,Wissens- und Innovationsgemeinschaften'; strategische Kontrolle der Wissensportfolios.

Ein Exekutivausschuss, der aus fünf Mitgliedern besteht, wobei eines der Mitglieder der Vorsitzende des Verwaltungsrates ist. Die übrigen vier Mitglieder werden vom Verwaltungsrat aus den Reihen der ernannten Mitglieder ausgewählt. Der Exekutivausschuss beschließt mit der einfachen Mehrheit seiner Mitglieder, überwacht die Arbeit des ETI und trifft in der Zeit zwischen den Sitzungen des Verwaltungsrates die erforderlichen Entscheidungen. ${ }^{20}$

Ein Direktor, der vom Verwaltungsrat für eine Amtsperiode von vier Jahren ernannt wird, die einmal verlängert werden kann. Der Direktor ist für die Geschäftsführung des ETI verantwortlich und ist dessen rechtlicher Vertreter. Hinsichtlich seiner Rechnungsführung ist er dem Verwaltungsrat gegenüber rechenschaftspflichtig. ${ }^{21}$

Ein Prüfungsausschuss, der fünf Personen umfasst, die vom Verwaltungsrat nach Konsultationen mit der Kommission aus den Reihen externer Berater mit angemessener Fachkompetenz für die Prüfung und Finanzkontrolle von Hochschul- und Forschungseinrichtungen sowie Unternehmen ausgewählt werden. Der Prüfungsausschuss berät den Verwaltungsrat und den Direktor in folgenden Angelegenheiten: Verwaltung und Finanzmanagement, Kontrollstrukturen innerhalb des ETI, Organisation der finanziellen Beziehungen zu den KIC sowie alle sonstigen Fragen, mit denen der Ausschuss vom Verwaltungsrat betraut wird. ${ }^{22}$

\section{Humanressourcen und Budget}

Um seine Exzellenzziele zu erreichen, muss das ETI sowohl auf die Einstellung hochqualifizierter Mitarbeiter als auch auf eine effiziente Arbeitsorganisation achten. In diesem Zusammenhang sind mehrere Konfigurationen für das Statut des Personals und der ,Wissensund Innovationsgemeinschaften 'vorgesehen: zum einen eine direkte personalrechtliche Einstellung, zum anderen aber auch eine Doppelzugehörigkeit, wobei der betreffende Mitarbeiter trotz Eingliederung in die Gasteinrichtung seinen ,ursprünglichen' Arbeitsplatz beibehält. Ebenso könnte es aber auch eine befristete Zuordnung zum Beispiel in Form von Sabbatjahren geben, ebenso wie auch eine ,Delegation ' beziehungsweise ein ,secondment", das heißt eine befristete Abstellung eines Mitarbeiters zu einer anderen Einrichtung. Unabhängig von der Wahl einer dieser verschiedenen dienstrechtlichen Formen müsste jedoch ein gemeinsamer Beschäftigungsrahmen festgelegt werden.

Beim ETI selbst wird nur eine sehr begrenzte Zahl von Personen beschäftigt sein. Wenn das ETI voll arbeitsfähig ist, wird es über höchstens 60 Bedienstete - zur Hälfte Wissenschaftler mit beratender Funktion und zur Hälfte Mitarbeiter für unterstützende Aufgaben - verfügen, die direkt vom ETI im Rahmen befristeter Verträge gemäß den Beschäftigungsbedingungen für die sonstigen Bediensteten der Europäischen Gemeinschaften ${ }^{23}$ angestellt werden.

20 Art. 8 Abs. 1 lit. b) Verordnungs-Vorschlag in Verbindung mit Art. 4 Satzung.

21 Art. 8 Abs. 1 lit. c) Verordnungs-Vorschlag in Verbindung mit Art. 5 Satzung.

22 Art. 8 Abs. 1 lit. d) Verordnungs-Vorschlag in Verbindung mit Art. 6 Satzung.

23 VO 259/68, Amtsblatt 1968, Nr. L 56, S. 1 ff. 
Der überwiegende Teil der Ausgaben des ETI wird zweifellos auf die ,Wissens- und Innovationsgemeinschaften " entfallen. In der Aufbauphase dürfte die Grundfinanzierung zum großen Teil aus öffentlichen Mitteln erfolgen. Im Zuge der Weiterentwicklung der ,Wissens- und Innovationsgemeinschaften " dürften dem ETI jedoch auch Mittel aus anderen gemeinschaftlichen oder nationalen Finanzierungsquellen, von Unternehmen, Stiftungen oder auch aus Studiengebühren zufließen. Eine öffentliche Bezuschussung bleibt jedoch unabdingbar. Die finanziellen Zuwendungen der Privatwirtschaft können dabei dreierlei Formen annehmen: zum einen die Bereitstellung von (finanziellen) Ressourcen durch private Unternehmen oder andere Partner, zum anderen die Erteilung von Aufträgen durch Privatunternehmen sowie auch die Gründung einer privaten Stiftung durch das ETI zur Einwerbung von Mitteln.

Die Gesamtausgaben des ETI und der ,Wissens- und Innovationsgemeinschaften ' werden von der Kommission für den Zeitraum 2007-2013 auf schätzungsweise 2,367,1 Millionen Euro veranschlagt. ${ }^{24}$ Gemäß Art. 16 des Verordnungs-Vorschlags beläuft sich der von der Haushaltsbehörde der EU veranschlagte Finanzrahmen auf 308,7 Millionen Euro. Ganz grundsätzlich wird das ETI Zugang zu Finanzmitteln des Siebten Forschungsrahmenprogramms (2007-2013) erhalten. In diesem Zusammenhang muss aber angemerkt werden, dass nur 15 Prozent der EU-Fördermittel für die Wissenschaft - beispielsweise über den Europäischen Forschungsrat - nach rein wissenschaftlichen Kriterien vergeben werden, während es bei den restlichen 85 Prozent nicht nur auf Qualität, sondern auch auf andere Maßstäbe, wie zum Beispiel die Regionalförderung oder die soziale Kohäsion, ankommt. ${ }^{25}$

\section{Der ,Europäische Forschungsrat"}

Das Programm ,Ideen`im Siebten Forschungsrahmenprogramm der EG (2007-2013) als Initialzündung

Nach einer intensiven Diskussion zwischen europäischen Wissenschaftlern über die Überwindung der Fragmentierung der wissenschaftlichen Forschung und der Erreichung eines einheitlichen ,Europäischen Forschungsraums` ergriffen am 26. November 2002 die europäischen Forschungsminister die Initiative und riefen die Europäische Kommission und die Mitgliedstaaten dazu auf, die Grundlagen eines europaweiten Forschungsrates auszuarbeiten. Der damalige dänische Ratspräsident, Helge Sander, lud kurz danach Prof. Federico Mayor, den früheren Generaldirektor der UNESCO (1987-1999) und heutigen Leiter der 2000 gegründeten „Fundación Cultura de Paz“ in Madrid, dazu ein, eine kleine Expertengruppe (ERCEG) einzuberufen, die eine entsprechende Machbarkeitsstudie erstellen sollte. Bereits im Dezember 2003 legte die Expertengruppe ihren Bericht ${ }^{26}$ vor, der konkrete Empfehlungen für die Einrichtung eines solchen Forschungsrates enthielt. Innerhalb kürzester Zeit erhielten diese Anregungen breite Zustimmung und zwar nicht nur von wissenschaftlicher sondern auch von politischer Seite.

Nachdem die Europäische Kommission im April 2005 die Grundzüge für das Siebte Forschungsrahmenprogramm (2007-2013) vorgelegt hatte, gestaltete sie dieses in der Folge näher aus und legte, wie vorstehend bereits erwähnt, am 21. September 2005 die vier wichtigsten spezifischen Programme vor, die das Rahmenprogramm spezieller ausgestalten sollen:

$24 \operatorname{SEK}(2006) 1313$ (Fn. 16), S. 10.

25 „Es geht nur um gute Wissenschaft“, in: Der Tagesspiegel, 27.02.2007.

26 Danish Ministry of Science, Technology and Innovation (Hrsg.): The European Research Council. A Cornerstone in the European Research Area, 15.12.2003, abrufbar unter: http://www.academie-sciences.fr/conferences/seances_publiques/pdf/Mayor_19_12_03.pdf (letzter Zugriff: 12.03.2007). 
- Das Programm ,Zusammenarbeit‘ soll es ermöglichen, eine führende Stellung in wissenschaftlichen und technologischen Schlüsselbereichen einzunehmen. Zu diesem Zweck wird die Zusammenarbeit zwischen Universitäten, Industrie, Forschungszentren und Behörden in der gesamten EU sowie dem Rest der Welt unterstützt. Die dabei vorgeschlagenen neun Themen ${ }^{27}$ entsprechen den für den wissenschaftlich-technologischen Fortschritt wichtigsten Gebieten, in denen die Spitzenforschung gestärkt werden muss, damit Europa seinen gesellschaftlichen, wirtschaftlichen, ökologischen und industriellen Herausforderungen gewachsen ist.

- Mit dem Programm ,Ideen ' soll ein Europäischer Forschungsrat zur europaweiten Förderung kreativer Wissenschaftler, Ingenieure und Akademiker eingesetzt werden, deren Neugier und Wissensdurst unvorhersehbare, umwälzende Entdeckungen möglich machen können, die unser Verständnis der Welt ändern, neue Aussichten für technologische Fortschritte eröffnen und eventuelle Lösungen für dauerhafte soziale und ökologische Probleme bieten können (sogenannte ,Pionierforschung ').

- Ziel des Programms ,Menschen“ ist die qualitative und quantitative Stärkung des europaweiten Humanpotenzials in Wissenschaft und Forschung. Es soll Anreize bieten, die Forschungslaufbahn einzuschlagen und zu durchlaufen, Forscher ermutigen, in Europa zu bleiben und die besten Köpfe dazu bewegen, nach Europa zu kommen.

- Das Programm ,Kapazitäten“ dient der Verbesserung der Forschungs- und Innovationskapazitäten in ganz Europa. Hierzu gehören neue Forschungsinfrastrukturen, die Förderung von kleinen und mittleren Unternehmen (KMU), die Entwicklung von wissensorientierten Regionen, die Ausschöpfung des Forschungspotenzials in Konvergenzregionen und in Regionen in äußerster Randlage sowie die bessere Verzahnung von Wissenschaft und Gesellschaft.

Definitiv wurde das „Siebte Rahmenprogramm der EG für Forschung, technologische Entwicklung und Demonstration“ (2007-2013) durch Beschluss Nr. 1982/2006/EG des Europäischen Parlaments und des Rates vom 18. Dezember $2006{ }^{28}$ und dessen spezifisches Programm „Ideen“ zu seiner Durchführung durch die Entscheidung 2006/972/EG des Rates vom 19. Dezember $2006^{29}$ eingerichtet. Art. 4 der Entscheidung 2006/972/EG sieht die Einrichtung eines Europäischen Forschungsrates durch die Kommission vor, der aus einem unabhängigen ,Wissenschaftlichen Rat' bestehen soll, der von einer spezifischen Durchführungsstelle unterstützt wird. ,Herzstück' des Forschungsrates ist damit ein ,Wissenschaftlicher Rat', der nach den Grundsätzen der wissenschaftlichen Exzellenz, Autonomie, Effizienz und Transparenz arbeiten und zunächst eingerichtet werden sollte.

\section{Die Auswahl des, Wissenschaftlichen Rates ‘}

Bereits Ende 2004 setzte der in der Europäischen Kommission für Wissenschaft und Forschung zuständige Kommissar Janez Potočnik einen unabhängigen Auswahlausschuss für die Nominierung der 22 Mitglieder des ,Wissenschaftlichen Rates' des geplanten Europäischen Forschungsrates ein, der unter dem Vorsitz des ehemaligen Kommissars für Auswärtige Beziehungen, Chris Patten - der inzwischen zum Chancellor of Oxford and Newcastleupon-Tyne Universities bestellt worden war - stehen und aus folgenden Personen bestehen

27 Gesundheit, Lebensmittel, Landwirtschaft und Biotechnologie, Informations- und Kommunikationstechnologien, Nanowissenschaften, Nanotechnologien, Werkstoffe und neue Produktionstechnologien, Energie, Umwelt (einschließlich Klimawandel), Sozial-, Wirtschafts- und Geisteswissenschaften, Sicherheit und Weltraum.

28 Amtsblatt 2006, Nr L 412, S. 1-2.

29 Amtsblatt 2006, Nr. L 400, S. 242. 
sollte: Prof. Erwin Neher (Nobelpreisträger, Max-Planck-Institut für biophysikalische Chemie, Göttingen), Dr. Catherine Bréchignac (Direktorin des Institut d’Optique, Université Paris Sud), Prof. Jüri Engelbrecht (Vizepräsident der Estnischen Akademie der Wissenschaften) und Prof. Guido Martinotti (Facoltà di Sociologia Università degli Studi di MilanoBicocca).

Der Auswahlausschuss nahm am 25. Januar 2005 seine Tätigkeit auf ${ }^{30}$, konnte aber erst Anfang Juli eine entsprechende Namensliste vorlegen, deren Personen vom EU-Forschungskommissar Janez Potočnik am 18. Juli 2005 in ihrer persönlichen Eigenschaft als Mitglieder des ,Wissenschaftlichen Rates“ des Europäischen Forschungsrates ernannt wurden. Dieser umfasst demgemäß folgende 22 hochqualifizierte und bekannte Wissenschaftlerinnen und Wissenschaftler: ${ }^{31}$

Dr. Claudio Bordignon (IT), San Raffaele Scientific Institute, Mailand

Prof. Manuel Castells (ES), Offene Universität von Katalonien, Barcelona, und University of California, Berkeley

Prof. Dr. Paul J. Crutzen (NL), Max-Planck-Institut für Chemie, Mainz

Prof. Mathias Dewatripont (BE), Université Libre de Bruxelles

Dr. Daniel Estève (FR), CEA Saclay

Prof. Pavel Exner (CZ), Doppler Institut, Prag

Prof. Dr. Hans-Joachim Freund (DE), Fritz-Haber-Institut, Berlin

Prof. Wendy Hall (UK), University of Southampton

Prof. Dr. Carl-Henrik Heldin (SE), Ludwig-Institut für Krebsforschung

Prof. Dr. Fotis C. Kafatos (GR), Imperial College London

Prof. Dr. Michael Kleiber (PL), Polnische Akademie der Wissenschaften

Prof. Norbert Kroo (HU), Ungarische Akademie der Wissenschaften

Prof. Maria Teresa V. T. Lago (PT), Universität Porto

Dr. Oscar Marin Parra (ES), Instituto de Neurociencias de Alicante

Prof. Lord May (UK), University of Oxford

Prof. Helga Nowotny (AT), Wissenschaftszentrum, Wien

Prof. Christiane Nüsslein-Volhard (DE), Max-Planck-Institut für Entwicklungsbiologie, Tübingen

Dr. Leena Peltonen-Palotie (FI), Universität Helsinki und National Public Health Institute Prof. Alain Peyraube (FR), CNRS, Paris

Dr. Jens R. Rostrup-Nielsen (DK), Haldor Topsoe A/S

Prof. Salvatore Settis (IT), Scuola Normale Superiore, Pisa

Prof. Dr. med. Rolf M. Zinkernagel (CH), Universität Zürich. ${ }^{32}$

Der ,Wissenschaftliche Rat" trat am 18./19. Oktober 2005 - im Beisein des Kommissars für Wissenschaft und Forschung - zu seiner ersten Sitzung zusammen ${ }^{33}$ und wählte auf einer weiteren Sitzung am 12. Dezember 2005 einstimmig Prof. Fotis Kafatos zu seinem Vorsitzenden und Prof. Helga Nowotny sowie Dr. Daniel Estève zu dessen beiden Vize-Vorsitzenden. Er regte in diesem Zusammenhang auch die Ernennung eines Generalsekretärs an, die zwar am 30. August 2006 stattfand, aber in einem Splitting dieser Position für die ersten fünf Jahre endete: Prof. Ernst-Ludwig Winnacker, Professor für Biochemie an der Universität München und Präsident der Deutschen Forschungsgemeinschaft (DFG), wird die Position

30 Vorbereitungen für einen Europäischen Forschungsrat, IP/05/90, 25.01.2005.

31 Europäischer Forschungsrat: Mitglieder des wissenschaftlichen Rates benannt, IP/05/956, 18.07.2005.

32 Biographies of Members of the Scientific Council, MEMO/05/265, 18.07.2005.

33 EU-Kommissar Potočnik kommt mit neuem wissenschaftlichen Rat zusammen zu seiner ersten Sitzung, IP/05/ 1297, 18.10.2005. 
des Generalsekretärs für den Zeitraum von Anfang Januar 2007 bis Ende Juni 2009, und Prof. Andreu Mas-Colell, Professor für Nationalökonomie an der Universität Pompeu Fabra in Barcelona, für die Periode von Anfang Juli 2009 bis Ende 2011 einnehmen. ${ }^{34}$ Auf die Frage an Herrn Winnacker, ob diese hälftige Teilung seiner Amtszeit nicht einen typischen ,EU-Kompromiss“ darstelle, erwiderte dieser, „,dass ich für die Aufbauarbeit geholt worden bin, und die ist Mitte 2009 abgeschlossen. Dann sind die Weichen gestellt“ “.35

\section{Die Konstituierung des Europäischen Forschungsrates}

Nach der Konstituierung des, Wissenschaftlichen Rates ‘ und der durch diesen vorgenommenen Wahl des Generalsekretärs war die Zeit reif für die Schaffung des ,Europäischen Forschungsrates`selbst. Am 18. Dezember 2006 stimmte der Rat der EU, nachdem das Europäische Parlament bereits früher sein Einverständnis bekundet hatte, der Errichtung des Europäischen Forschungsrates und seinem Sieben-Jahres-Budget in Höhe von 7,5 Milliarden Euro (politisch) zu, es kam aber erst am 2. Februar 2007 zur definitiven Errichtung des Europäischen Forschungsrates (EFR/ERC). Gemäß Art. 1 des Beschlusses 2007/134/EG der Kommission zur Errichtung des Europäischen Forschungsrates ${ }^{36}$ wurde der Europäische Forschungsrat für den Zeitraum vom 2. Februar 2007 bis zum 31. Dezember 2013 zur Durchführung des speziellen Programms „Ideen“37 eingerichtet.

Die offizielle Gründung des Europäischen Forschungsrates wurde allerdings erst am 27./ 28. Februar 2007 in Berlin im Rahmen einer Konferenz vorgenommen, die vom deutschen Bundesministerium für Bildung und Forschung und von der Deutschen Forschungsgemeinschaft (DFG) gemeinsam organisiert wurde. An ihr nahmen die deutsche Bundeskanzlerin und Ratsvorsitzende, Angela Merkel, der EU-Forschungskommissar Janez Potočnik, der Präsident des Europäischen Forschungsrates, Fotis Kafatos, Mitglieder des Wissenschaftlichen Beirates und weitere Vertreter der europäischen ,scientific community " teil. Der Sitz des Forschungsrates befindet sich in Brüssel.

\section{Die administrative Struktur des ,Europäischen Forschungsrates ‘}

Gemäß Art. 1 des Beschlusses 2007/134/EG der Kommission besteht der Europäische Forschungsrat aus einem ,Wissenschaftlichen Rat' und einer , spezifischen Durchführungsstelle'.

Gemäß Art. 2 dieses Beschlusses wurde der bereits provisorisch errichtete ,Wissenschaftliche Rat' ebenfalls definitiv eingerichtet und gemäß Art. 3 mit den in Art. 5 Abs. 3 der vorerwähnten Entscheidung 2006/972/EG ${ }^{38}$ genannten Aufgaben betraut. Gemäß Art. 4 Abs. 3 des Beschlusses 2007/134/EG werden auch die auf der Grundlage der in dessen Anhang I dargelegten Kriterien benannten und bereits provisorisch bestellten 22 Gründungsmitglieder - die in Anhang II nominatim aufgeführt sind - definitiv berufen. Die Amtsdauer der Mitglieder des Wissenschaftlichen Rates beträgt gemäß Art. 4 Abs. 6 vier Jahre. Gemäß Art. 5 arbeitet der Wissenschaftliche Rat autonom und unabhängig und nur im Interesse der wissenschaftlichen, technologischen und akademischen Ziele des spezifischen Programms ,Ideen“ des Siebten Rahmenprogramms (2007-2013). Gemäß Art. 6 wählt er einen Präsidenten beziehungsweise Vizepräsidenten, was - wie vorstehend bereits erwähnt - bereits am 12 .

34 ERC: ERC Secretary General announced, Presseerklärung, 30.08.2006.

35 „Es geht nur um gute Wissenschaft“, in: Der Tagesspiegel, 27.02.2007.

36 Amtsblatt 2007, Nr. L 57, S. 14 ff.

37 Ebenda.

38 Ebenda. 
Dezember 2005 geschehen ist. Die in Art. 7 vorgesehene Wahl eines Generalsekretärs des Wissenschaftlichen Rates ist ebenfalls bereits vorgenommen worden.

Obwohl bisher in seiner 14-monatigen Aufbauphase lediglich informell tätig, legte der ,Wissenschaftliche Rat‘ am 2. Februar 2007 seinen „Report of the ERC Scientific Council on the occasion of its formal establishment " 39 vor und ersuchte die Kommission, diesen sowohl dem Europäischen Parlament als auch dem Rat weiterzuleiten.

Gemäß Art. 9 des Beschlusses 2007/134/EG wird die ,spezifische Durchführungsstelle“ als externe Stelle eingerichtet. Bis diese externe Stelle gegründet und einsatzfähig ist, werden ihre Durchführungsaufgaben von einer besonderen Dienststelle der Europäischen Kommission - GD Forschung, Direktion S-Implementierung der ,Ideen'-Programme der Europäischen Kommission (,Dedicated Implementation Structure') - wahrgenommen.

Bis Mitte 2008 soll die externe Stelle dann in Form einer ,Exekutiv-Agentur' eingerichtet werden, bei der es sich um ein Instrument zur „Externalisierung “40 - im Sinne der Auslagerung spezieller Verwaltungsaufgaben der Kommission auf selbständige Verwaltungseinheiten - in Form einer sogenannten „Dekonzentration“41 handelt. Dabei kommt es zu einer Übertragung von Exekutivaufgaben an öffentliche Einrichtungen in der Gemeinschaft mit eigener Rechtspersönlichkeit, die unter der Kontrolle und Verantwortung der Kommission damit beauftragt werden, ,Gemeinschaftsprogramme ‘ im Sinne von Art. 2 lit. b) der Verordnung (EG) Nr. 58/2003 des Rates vom 19. Dezember 2002 zur Festlegung des Statuts der Exekutivagenturen, die mit bestimmten Aufgaben bei der Verwaltung von Gemeinschaftsprogrammen beauftragt werden, ${ }^{42}$ durchzuführen und zu verwalten. Durch die Verordnung (EG) Nr. 58/2003 wurde ein eigenes ,Musterstatut' für Exekutivagenturen aufgestellt, das die Struktur, die Leitung, das Personal, die Zuständigkeiten und Aufgaben, die Arbeitsweise, die Beziehungen zur Kommission, die Haushalts- und Finanzstrukturen, die Kontrolle, die rechtliche und finanzielle Haftung einer ,Exekutivagentur' detailliert regelt. ${ }^{43}$

\section{Vergabe der Fördermittel}

Was den Schlüssel für die Verteilung der Fördermittel betrifft, so wurde dem Europäischen Forschungsrat statutarisch kein eigener Verteilungsschlüssel vorgegeben. Der Wissenschaftliche Rat orientierte sich diesbezüglich an den Mittelwerten sonstiger Förderorganisationen und legte intern folgenden indikativen Schlüssel fest: 15 Prozent der Mittel gehen in die Geistes- und Sozialwissenschaften, 45 Prozent in die Natur- und Ingenieurwissenschaften und 40 Prozent in die Lebenswissenschaften und die Medizin. Da der Wert für die Geisteswissenschaften in den einzelnen Fonds zwischen 10 und 20 Prozent schwankt, hat sich der Forschungsrat letztlich für den Mittelwert von 15 Prozent entschieden. ${ }^{44}$ Vom Gesamtbudget soll eine 20-prozentige Reserve zurückgestellt werden, damit einmal genehmigte Anträge auch weiter unterstützt werden können, obwohl bei ihnen die maximale Förderhöhe bereits überschritten wurde.

39 Siehe Europäischer Forschungsrat: http://erc.europa.eu (letzter Zugriff: 12.03.2007).

40 Vgl. dazu die Mitteilung von Frau Schreyer und Vizepräsident Kinnock, SEK (2000) 2051 vom 14. Dezember 1999, S. 7, in der eigene ,Leitlinien“ für die Externalisierungspolitik der Kommission festgelegt wurden.

41 Vgl. Waldemar Hummer: Von der „Agentur“ zum „Interinstitutionellen Amt“, in: Stefan Hammer/Alexander Somek/Manfred Stelzer/BarbaraWeichselbaum (Hrsg.): Demokratie und sozialer Rechtsstaat in Europa, Festschrift für Theo Öhlinger, Wien 2004, S. 92-130, hier S. 115.

42 Amtsblatt, 2003, Nr. L 11, S. 1 ff.

43 Vgl. Waldemar Hummer: Von der „Agentur“ zum „Interinstitutionellen Amt“, S. 116.

44 Europäischer Forschungsrat: The IDEAS (Provisional) Work Programme. European Research Council Work Programme (2006); „Es geht nur um gute Wissenschaft“, in: Der Tagesspiegel, 27.02.2007. 
Für die Bewerbung um die Fördermittel des EFR spielt die Nationalität der Bewerber keine Rolle, entscheidend ist nur, dass die Forschungsleistung in einem EU-Mitgliedsland oder einem mit der EU assoziierten Land beziehungsweise auch in einem Land mit dem Status eines Beitrittskandidaten erbracht wird. Es kann sich also zum Beispiel ein Argentinier bewerben, der in Deutschland forschen will, oder auch ein Deutscher, der gegenwärtig in den USA forscht und an eine britische Universität wechseln will. Die wissenschaftliche Einrichtung, an der der Antragsteller tätig werden will, muss sich dementsprechend nur in einem EU-Mitgliedstaat, einem mit der EU assoziierten Staat oder einem Land mit Kandidatenstatus befinden.

Die Fördermittel werden formell zwar den Einrichtungen angewiesen, die den ausgewählten hauptverantwortlichen Forscher beherbergen, diese müssen sich aber verpflichten, diesem Wissenschaftler all diejenigen Freiheiten zu gewähren, die dieser für die ordnungsgemäße Durchführung des von ihm beantragten Projekts benötigt. Damit trifft die Institute und Universitäten, an denen diese handverlesenen Wissenschaftler arbeiten wollen, eine Reihe von speziellen Verpflichtungen: sie müssen zum einen ein gutes akademisches Umfeld bieten, das heißt vor allem Doktoranden bereitstellen, gute Arbeitsbedingungen garantieren, ein unabhängiges Publizieren ermöglichen und auch ein entsprechendes Gehalt zahlen.

Die Fördermittel werden in zwei Gruppen eingeteilt, nämlich zum einen in die ,ECR Starting Independent Researcher Grants ‘ und zum anderen in die ,ECR Advanced Investigator Grants', die beide nach dem ,bottom-up'- oder , investigator-driven'-System ohne irgendwelche vorgegebenen Prioritäten arbeiten und Exzellenz-Projekte unterstützen sollen, die von individuellen Teams, die unter der Leitung eines hauptverantwortlichen Forschers stehen, beantragt werden. Mit dieser Schwerpunktsetzung der Unterstützung eines hauptverantwortlichen Forschers samt seinem Team unterscheidet sich die EFR-Förderung grundlegend von sonstigen ,Netzwerken“ oder ,Forscher-Konsortien', die zum Beispiel vom vorerwähnten Europäischen Technologieinstitut (ETI) unterstützt werden.

Was die ,ECR Starting Independent Researcher Grants ‘ betrifft, so sind sie für hervorragende Nachwuchswissenschaftler (Principal Investigators) vorgesehen, die bereits fähig sind, ein eigenes Forschungsteam zu leiten. Der Nachwuchsforscher muss dabei eigenständig einen solchen Förderungsantrag stellen können, ohne dabei auf die Mitwirkung eines erfahreneren Kollegen angewiesen zu sein. Er muss auch schon eigenständig publiziert haben und in der Lage sein, als gleichberechtigter Ko-Autor mit Kollegen zu publizieren. In der Regel geht es dabei um Wissenschaftler, die sich in einem Alter zwischen zwei und neun Jahren nach ihrer Promotion (postdoc) befinden. Insgesamt dürfen aber nicht mehr als 12 Jahre seit der Verleihung des Doktorates verstrichen sein. Am Ende der Förderperiode sollen die Principal Investigators den Übergang zur völligen wissenschaftlichen Selbständigkeit geschafft haben. ${ }^{45}$

Was hingegen die ,ECR Advanced Investigator Grants ‘ betrifft, so sollen sie - in Ergänzung zu den Förderungen von Nachwuchswissenschaftlern - bereits arrivierte Spitzenforscher in die Lage versetzen, herausragende wissenschaftliche Projekte durchzuführen.

45 Für die Kriterien dieser Selbständigkeit siehe: The European Charter for Researchers and The Code of Conduct for the Recruitment of Researchers, abrufbar unter: http://ec.europa.eu/eracareers/pdf/am509774CEE_EN_ E4.pdf (letzter Zugriff: 12.03.2007). 


\section{Umfang der Förderung}

Insgesamt stehen im Rahmen des Europäischen Forschungsrates Förderungsmittel in Höhe von 7,5 Milliarden Euro für den siebenjährigen Zeitraum von 2007 bis 2013 zur Verfügung, wobei im Startjahr 2007 zunächst rund 850 Millionen Euro zur Ausschüttung kommen werden. Ab dem Jahr 2008 werden dann jährliche Tranchen von circa 1 Milliarde Euro zur Auszahlung kommen.

Die Höhe der Förderbeträge für Principal Investigators beträgt dabei rund 300 Millionen Euro, was mehr als einem Drittel des Jahresbudgets entspricht. Der Förderbetrag für einen einzelnen Nachwuchswissenschaftler liegt zwischen 100.000 Euro und 400.000 Euro pro Jahr für eine maximal fünf-jährige Periode, sodass ein Principal Investigator maximal mit 2 Millionen Euro gefördert werden kann. Die gemeinschaftsrechtliche Komponente zu diesem Förderprogramm besteht in einem finanziellen Zuschuss in Höhe von 100 Prozent der gesamten zuteilbaren und bewilligten direkten Kosten sowie in einem 20-prozentigen Zuschuss - unter Ausschluss eventueller Subkontrahierungen - zu den ,overheads“ im Sinne von indirekten Kosten. Die tatsächliche Förderhöhe wird durch das Ergebnis der , peer review evaluation" der jeweils beantragten Fördersumme determiniert.

Für renommierte, ältere Wissenschaftler stehen anlässlich der ersten Ausschreibung in der zweiten Hälfte 2007 insgesamt rund 550 Millionen Euro zur Verfügung, die allerdings erst 2008 zur Auszahlung kommen werden.

\section{Stellung und Bewertung des Förderantrags}

Förderanträge sind vom Principal Investigator im Namen der wissenschaftlichen Einrichtung, der er angehört (,applicant legal entity'), zu stellen, wobei letztere sich zu verpflichten hat, ihm den entsprechenden wissenschaftlichen Freiraum für seine Forschungen zu gewähren. Die Anträge können sich dabei auf jeden Bereich wissenschaftlicher Forschung beziehen und können beziehungsweise sollen sogar interdisziplinär und grenzüberschreitend sein. Auch sogenannte ,high-risk, high-gain'-Anträge sind zulässig. EFR-Anträge unterscheiden sich damit ganz grundlegend von sogenannten , postdoctoral grants'. Projekte zur Stammzellenforschung können nur innerhalb des ,Ethischen Rahmens ', wie er im Siebten Forschungsrahmenprogramm (2007-2013) formuliert ist, ${ }^{46}$ eingereicht werden, sodass Projekte, die die Tötung von Embryonen zur Stammzellengewinnung vorsehen, nicht gefördert werden können.

Was die Beantragung der Fördermittel betrifft, so ist dafür ein zweistufiges Verfahren vorgesehen. In einer ersten Phase wird eine kurze Projektbeschreibung, zusammen mit den bisherigen wissenschaftlichen Meriten des Antragstellers und einer Beschreibung des wissenschaftlichen Umfelds, erbeten, die anschließend evaluiert wird. Diejenigen Antragsteller, die diese Phase positiv abschließen können, werden eingeladen, sich für die zweite Auswahlphase - nunmehr aber mit einem ausführlichen Antrag - zu bewerben. Was den Umfang der Förderanträge betrifft, so darf er in der ersten Phase (,Outline proposal') acht Seiten und in der zweiten (,Full proposal“) 16 Seiten nicht überschreiten. In der ersten Phase muss die wissenschaftliche Einrichtung, der der Antragsteller angehört, ihre Bereitschaft erklären, das Projekt zu unterstützen und zu fördern, und in der zweiten Phase hat sie zusätzlich die vorerwähnte Erklärung abzugeben, dass sie dem Wissenschaftler den notwendigen Freiraum für seine Forschungen lässt.

46 Vgl. dazu die Erklärung der Kommission im Annex IV der Politischen Übereinkunft des Europäischen Rates über das Siebte Forschungsrahmenprogramm (2007-2013) vom 25. Juli 2006; für ein ähnliches Problem im Sechsten Forschungsrahmenprogramm (2002-2006) siehe Albin Christoph Lohninger: Interdisziplinäre, völker- und europarechtliche Grundlagen der Gen- und Biotechnologie, Baden-Baden 2007, S. 332-333. 
Am 22. Dezember 2006 kündigte die Kommission ihren ersten Aufruf für die Einreichung von Anträgen zur Gewährung von ,Start-Stipendien“ an, der in der Folge auf der Sitzung zur formellen Errichtung des ,Wissenschaftlichen Rates'vom 2. Februar 2007 verlautbart wurde. Für die ,ECR Starting Independent Researcher Grants' können die Anträge nunmehr elektronisch - ausschließlich mittels des web-basierten ,Electronic Proposal Submission System ‘ (EPSS) ${ }^{47}$ - ab dem 18. März 2007 eingereicht werden, wobei die Einreichfrist bereits am 25. April 2007 endet. Dabei ist eine ,pre-registration' notwendig, um ein Login und Passwort für EPSS zu erhalten. ${ }^{48}$ Zunächst werden die ,outline proposals ' von einem fachspezifischen Review-Panel begutachtet. Danach wird eine Auswahl von Kandidaten eingeladen, ein ,full proposal' einzureichen. Dieses wird von externen Gutachtern sowie von den Mitgliedern des Review Panels begutachtet. Danach werden die bestplatzierten Kandidaten zu Interviews eingeladen. Über die endgültige Vergabe entscheidet das gesamte Review Panel. ${ }^{49}$ In Summe werden an diesem Auswahlprozess über 20 Review Panels mit je zehn Personen beteiligt sein. Im November 2007 wird dann die endgültige Entscheidung über die Zuerkennung von Fördermitteln fallen, wobei rund 200 Förderanträge bewilligt werden.

Der Startschuss für die Einreichung der Anträge für die ,ECR Advanced Investigator Grants ' wird in der zweiten Hälfte des Jahres 2007 - im Rahmen der zweiten Runde der Antragstellung für die ,ECR Starting Independent Researcher Grants ' erfolgen. Ab dem Jahr 2008 werden dann aber beide Fördertypen mit einem Volumen von einer Milliarde Euro pro Jahr parallel ausgeschrieben.

Zur Information und Beratung der Antragsteller für Stipendien des Europäischen Forschungsrates wurden auch ,Nationale Kontakt-Stellen “ eingerichtet, die zum Beispiel in Österreich mit Ralf König von der Forschungsföderungs-Gesellschaft (FFG) und in der Bundesrepublik mit Christiane Wehle von der EU-Abteilung des Bundesministeriums für Bildung und Forschung besetzt sind.

\section{Koordinierungs- und Unterstützungsaktionen}

Zur Optimierung seiner gegenwärtigen Fördertätigkeit, zur Verbesserung seiner zukünftigen Aktivitäten und zur Verbreitung der gewonnenen Forschungsergebnisse fördert der Europäische Forschungsrat sogenannte ,Koordinierungs- und Unterstützungsaktionen ' (Coordination and Support Actions, CSAs), die gesondert ausgeschrieben werden. Solche Aktionen können auch zur Unterstützung des Generalsekretärs des EFR eingesetzt werden, falls dieser mit seiner eigenen administrativen Infrastruktur, dem Wissenschaftlichen Rat des EFR und der Kommission zwecks Monitoring und Evaluierung von Forschungsprojekten in engeren Kontakt treten muss. Die Förderung kommt in diesem Falle einer benannten Person zugute, die im Arbeitsprogramm der Europäischen Kommission für das Jahr 2007 spezifiziert wird. Das maximale Budget für solche ,Koordinierungs- und Unterstützungsaktionen“ ist mit 335.000 Euro für das Jahr 2007 festgesetzt. ${ }^{50}$

47 Detaillierte Informationen zur Antragstellung und zu EPSS finden sich im "Guide for Applicants" beziehungsweise im „EPSS preparation and submission guide“, abrufbar unter: http://cordis.europa.eu/fp7/dc/index.cfm?fuseaction=UserSite.InputFP7DetailsCallPage\&CALL_ID=3\#infopack (letzter Zugriff: 12.03.2007).

48 Europäischer Forschungsrat: First ECR call for Proposals; abrufbar unter: http://cordis.europa.eu/fp7/dc/index.cfm?fuseaction=page.display\&topicID=96 (letzter Zugriff: 12.03.2007).

49 In diesem Zusammenhang wird im Report of the ERC Scientific Council on the occasion of its formal establishment (02.02.2007), S. 3, expressis verbis darauf hingewiesen, dass ,the unprecedented acceptance rate approaching 95 percent of the persons invited to participate in the peer review evaluation panels for Starting Grants“ ein Beleg für den ,enthusiasm amongst Europe's scientists towards the ERC and the strategy of the Scientific Council" ist. 


\section{Schlussbetrachtungen}

In der Erkenntnis, dass die Innovations- und Technologiekluft, die zwischen Europa und den Vereinigten Staaten klafft, à la longue geschlossen werden muss und unter Berücksichtigung der alten und neuen Herausforderungen, die auf Europa seitens der asiatischen Staaten Japan, China und Indien zukommen, hat die Kommission zwei Instrumente entwickelt, mittels derer europäische Hochtechnologie zur industriellen Verwertung sowie akademische Spitzenforschung gefördert beziehungsweise überhaupt erst ermöglicht werden soll. Die beiden Instrumente, nämlich das Europäische Technologieinstitut (ETI) und der Europäische Forschungsrat (EFR/ERC), dienen zwar gleichzeitig beiden Zielsetzungen, versuchen diese aber auf unterschiedlichen Wegen zu erreichen.

Während das Europäische Technologieinstitut versucht, in Europa tätige Spitzenforscher miteinander in ,Wissens- und Innovationsgemeinschaften ${ }^{`}$ zu vernetzen, fördert der ,Europäische Forschungsrat' einzelne Forscher - und zwar sowohl hochqualifizierten wissenschaftlichen Nachwuchs als auch bereits etablierte Wissenschaftler - mit ihren Teams, um auf diese Weise Exzellenzzentren aufzubauen. Beide Ansätze sind damit komplementär konzipiert und ergänzen einander in synergetischer Weise.

Die Dotierung beider Instrumente - das ETI verfügt für die Periode 2007-2013 über ein Gesamtbudget von 2,367,1 Millionen Euro, davon werden 308,7 Millionen Euro aus dem Gemeinschaftshaushalt gezahlt, während der EFR/ERC in derselben Periode über einen mehr als dreimal so hohen Etat von 7,5 Milliarden Euro verfügt - erscheint zunächst ausreichend, um in ihren jeweiligen Zielsetzungen zumindest für eine Initialzündung zu sorgen. Entscheidend wird in der Folge aber sein, ob es in Europa - so wie es in den USA bereits seit Langem praktiziert wird - gelingt, die Industrie stärker in die Forschungsfinanzierung einzubinden. Allzu lange waren in Europa die beiden Bereiche Wissenschaft \& Forschung und Industrie voneinander unabhängig, und die wissenschaftliche Forschung verstand sich eher als akademische Grundlagenforschung denn als industrieorientierte Auftragsforschung. Falls es in den nächsten Jahren - und es ist diesbezüglich bereits ,fünf nach Zwölf' - nicht gelingt, diesen Trend zu stoppen oder sogar umzudrehen, wird Europa wissenschaftlich und technologisch in das zweite Glied zurücktreten, an Innovationskraft verlieren und in der Folge alle damit verbundenen sozio-ökonomischen Probleme zu bewältigen haben.

50 Europäischer Forschungsrat: The IDEAS. Work Programme. 